PROCEEDINGS OF THE

AMERICAN MATHEMATICAL SOCIETY

Volume 125, Number 12, December 1997, Pages 3735-3739

S 0002-9939(97)04190-7

\title{
HOMOTOPY FIXED POINTS FOR CYCLIC $p$-GROUP ACTIONS
}

\author{
W. G. DWYER AND J. M. MØLLER \\ (Communicated by Thomas Goodwillie)
}

\begin{abstract}
The homotopy fixed point $p$-compact groups for cyclic $p$-group actions on nonabelian connected $p$-compact groups are not homotopically discrete.
\end{abstract}

\section{INTRODUCTION}

It is a classical result that cyclic groups acting on nonabelian compact connected Lie groups have no isolated fixpoints [2, Lemme 1, p. 46]:

Theorem 1.1. Let $X$ be a nonabelian connected compact Lie group equipped with an action of a cyclic group $G$. Then the identity component of the fixed point group $X^{G}$ is nontrivial.

In this note we prove an analog for $p$-compact groups of this statement. First, we need a few concepts.

Suppose that $X$ is a $p$-compact group [4] with classifying space $B X$ and that $G$ is a finite group.

Definition 1.2. A $G$-action on $X$ is a sectioned fibration

$$
B X \longrightarrow(B X)_{h G} \underset{B a}{\rightleftarrows} B G
$$

over $B G$ with fibre $B X$.

If $G$ is a finite $p$-group, it is known $[4,5.8]$ that each component of the section space $(B X)^{h G}$ is the classifying space of a $p$-compact group. We define the homotopy fixed point p-compact group for the $G$-action to be the $p$-compact group

$$
X^{h G}=\Omega\left((B X)^{h G}, B a\right)
$$

whose classifying space is the component containing the section $B a$.

Having introduced these concepts, we can now formulate the main result of this note. (A connected $p$-compact group is nontrivial if its classyfying space is noncontractible.)

Theorem 1.3. Let $X$ be a nonabelian connected $p$-compact group equipped with an action of a cyclic p-group $G$. Then the identity component of the homotopy fixed point p-compact group $X^{h G}$ is nontrivial.

Received by the editors July 10, 1996.

1991 Mathematics Subject Classification. Primary 55P35.

Key words and phrases. Fixed point, $p$-compact group, Lefschetz number, Weyl group.

(C)1997 American Mathematical Society 
The following consequence of this theorem, whose proof relies on a Lefschetz number calculation, is immediate.

Corollary 1.4. Let $\nu: G \rightarrow Y$ be a monomorphism of a cyclic p-group into a (not necessarily connected) p-compact group $Y$ which is not a p-compact toral group. Then the identity component of the centralizer $C_{Y}(G)$ of $\nu$ is nontrivial.

The self-centralizing diagonal subgroup $(\mathbb{Z} / 2 \mathbb{Z})^{n}$ of $\mathrm{O}(n)$ shows that noncyclic subgroups may have discrete centralizers.

Corollary 1.4 plays an important role in the proof of the main result of [9].

\section{A Lefschetz number CALCUlation}

Let $X$ be a connected $p$-compact group, $G=\mathbb{Z} / p^{r}, r \geq 0$, a cyclic $p$-group and

$$
B X \longrightarrow(B X)_{h G} \underset{B a}{\rightleftarrows} B G
$$

an action of $G$ on $X$. The homotopy fixed point $p$-compact group $X^{h G}$ is the section space of the fibrewise looping

$$
X \longrightarrow X_{h G} \rightleftarrows B G
$$

of the $G$-action. Consider the associated monodromy homomorphisms

$$
\begin{gathered}
G \rightarrow \operatorname{Aut}_{*}(B X), \\
G \rightarrow \operatorname{Aut}_{*}(X)
\end{gathered}
$$

of $G$ into the groups of based homotopy classes of based self-homotopy equivalences of the fibres and the induced representations

$$
\begin{gathered}
G \rightarrow \operatorname{Aut} H_{\mathbb{Q}_{p}}^{*}(B X), \\
G \rightarrow \operatorname{Aut} H_{\mathbb{Q}_{p}}^{*}(X)
\end{gathered}
$$

of $G$ in the $p$-adic rational cohomology algebras. Of course, representation (4) induces yet another representation

$$
G \rightarrow Q H_{\mathbb{Q}_{p}}^{*}(B X)
$$

of $G$ in the graded vector space of indecomposables.

Let $B g: B X \rightarrow B X$ and $g: X \rightarrow X$ be the self-homotopy equivalences induced by a generator $g \in G$. We shall compute the Lefschetz number

$$
\Lambda(X ; G)=\sum(-1)^{i} \text { trace } H_{\mathbb{Q}_{p}}^{i}(g)
$$

for the action of $G$ on $X$ in terms of the irreducible summands of the representation (5).

Recall $[4, \S 4]$ that the cyclic group $G$ admits $r+1$ essentially distinct irreducible representations $\rho_{0}, \rho_{1}, \ldots, \rho_{r}$ over the $p$-adic numbers. Here, $\rho_{0}$ is the trivial representation and $\rho_{i}, 1 \leq i \leq r$, is the composition of the reduction map $G=\mathbb{Z} / p^{r} \rightarrow \mathbb{Z} / p^{i}$ with the action of $\mathbb{Z} / p^{i}$, regarded as the group of $p^{i}$ th roots of unity, on the extension field $\mathbb{Q}_{p}\left(\omega_{i}\right)$ of $\mathbb{Q}_{p}$ by a primitive $p^{i}$ th root of unity $\omega_{i}$. The dimension of $\rho_{i}, 1 \leq i \leq r$, is $\left[\mathbb{Q}_{p}\left(\omega_{i}\right): \mathbb{Q}_{p}\right]=p^{i}-p^{i-1}$. 
Proposition 2.1. Suppose that the G-representation $Q H_{\mathbb{Q}_{p}}^{*}(B X)$ contains the irreducible representation $\rho_{i}$ with multiplicity $n_{i}, 0 \leq i \leq r$. Then

$$
\Lambda(X ; G)= \begin{cases}p^{n_{1}+\cdots+n_{r}} & \text { if } n_{0}=0, \\ 0 & \text { if } n_{0} \neq 0\end{cases}
$$

is the Lefschetz number for the action of $G$ on $X$. In particular, $\Lambda(X ; G)=0$ if and only if $G$ fixes a nonzero vector of $Q H_{\mathbb{Q}_{p}}^{*}(B X)$.

Proof. Note that the monodromy action (2) of $G$ on $X=\Omega B X$ is the looping of the monodromy action (1) on $B X$ and that the Eilenberg-Moore spectral sequence provides a functorial isomorphism between the graded object $\operatorname{Gr}\left(H_{\mathbb{Q}_{p}}^{*}(X)\right)$ associated to a filtration of $H_{\mathbb{Q}_{p}}^{*}(X)$ and the exterior algebra $E\left(\Sigma^{-1} Q H_{\mathbb{Q}_{p}}^{*}(B X)\right)$ on the desuspension of $Q H_{\mathbb{Q}_{p}}^{*}(B X)$. Combining this with the isomorphism

$$
Q H_{\mathbb{Q}_{p}}^{*}(B X) \cong n_{0} \rho_{0} \oplus n_{1} \rho_{1} \oplus \cdots \oplus n_{r} \rho_{r}
$$

of $G$-representations induces yet another isomorphism

$$
\operatorname{Gr}\left(H_{\mathbb{Q}_{p}}^{*}(X)\right) \cong E\left(\Sigma^{-1} \rho_{0}\right)^{\otimes n_{0}} \otimes E\left(\Sigma^{-1} \rho_{1}\right)^{\otimes n_{1}} \otimes \cdots \otimes E\left(\Sigma^{-1} \rho_{r}\right)^{\otimes n_{r}}
$$

of $G$-representations. By the additivity [4, 4.12] of traces in exact sequences, then, the Lefschetz number

$$
\Lambda(X ; G)=\prod_{i=0}^{r} \Lambda_{i}^{n_{i}}
$$

where $\Lambda_{i}$ is the trace for the action of $G$ on $E\left(\Sigma^{-1} \rho_{i}\right)$.

Since $E\left(\Sigma^{-1} \rho_{0}\right)$ is the trivial representation, $\Lambda_{0}=0$.

When $i>0$, we pass to an algebraic closure of $\mathbb{Q}_{p}$. Then $\rho_{i}$ splits into 1dimensional representations and we see that $\Lambda_{i}=\Phi_{i}(1)$ where $\Phi_{i}$ is the characteristic polynomial for $g$ acting on $\rho_{i}$ or, equivalently, for $\omega_{i}$ acting on $\mathbb{Q}_{p}\left(\omega_{i}\right)$. Hence $\Phi_{i}$ is the $p^{i}$ th cyclotomic polynomial so $\Phi_{i}(1)=p$ and the proposition follows.

The consequence below is evident if we recall $[4,4.5,5.7,5.10]$ that the Lefschetz number $\Lambda(X ; G)$ computes the Euler characteristic of $X^{h G}$ and that a $p$-compact group is homotopically discrete if it looks so in $p$-adic rational cohomology.

Corollary 2.2. The following conditions are equivalent:

(1) $X^{h G}$ has a nontrivial identity component.

(2) $\chi\left(X^{h G}\right)>0$.

(3) $\Lambda(X ; G)>0$.

(4) $G$ fixes a nonzero vector of $Q H_{\mathbb{Q}_{p}}^{*}(B X)$.

The proof of Theorem 1.3 has now been reduced to the following

Lemma 2.3. Suppose that $X$ is nonabelian (i.e. not a p-compact torus). Then $G$ fixes a nonzero vector of $Q H_{\mathbb{Q}_{p}}^{*}(B X)$.

Proof. Let $T \rightarrow X$ be a maximal torus with Weyl group $W$. The dual weight lattice $L=\pi_{2}(B T)$ is then a $\mathbb{Z}_{p}[W]$-module whose rationalization $L \otimes \mathbb{Q}$ exhibits $W$ as a reflection group over $\mathbb{Q}_{p}$. The action of $G$ on the symmetric invariants $\operatorname{Sym}\left((L \otimes \mathbb{Q})^{*}\right)^{W} \cong H_{\mathbb{Q}_{p}}^{*}(B X)$ factors $[4,8.11,9.5],[8, \S 3]$ through $N(W) / W$ where $N(W)$ is the normalizer of $W<\operatorname{Aut}(L \otimes \mathbb{Q})$.

Suppose first that $X$ is almost simple, i.e. $[5,1.6]$ that the center of $X$ is finite and that $L \otimes \mathbb{Q}$ is a simple $\mathbb{Q}_{p}[W]$-module. Then the reflection group $W$ is one of the 
irreducible reflection groups on the Shephard-Todd-Clark-Ewing list as presented e.g. in $[6$, p. 165]. The list provides information about the indecomposables of the invariant ring in that the degrees of each reflection group are given.

If $p>2$, the list shows that $\operatorname{dim}_{\mathbb{Q}_{p}} Q H_{\mathbb{Q}_{p}}^{i}(B X)<p-1$ for all $i$. (In fact $Q H_{\mathbb{Q}_{p}}^{i}(B X)$ has dimension $\leq 2$ with dimension 2 occurring only in case 2 a (where the degrees given in [6] are incorrect) and in case 19, neither of which are realizable for $p=3$.) Since a nontrivial $p$-adic representation of a cyclic $p$-group requires at least $p-1$ dimensions, $G$ must act trivially on all of $Q H_{\mathbb{Q}_{p}}^{*}(B X)$ (which is nonzero if $X$ is nontrivial $[4,5.10])$.

The case $p=2$ requires separate treatment. The only irreducible 2-adic reflection groups are the classical Coxeter groups together with group number 24 of rank $3, W=\mathbb{Z} / 2 \mathbb{Z} \times \mathrm{GL}_{3}\left(\mathbb{F}_{2}\right)$, realized by $\mathrm{DI}(4)$ [3]. If $W$ is one of the classical Coxeter groups, the effect of an element of the normalizer $N(W)$ on the degree 4 invariants is multiplication by $u^{2}, 2 u^{2}$, or $3 u^{2}$, where $u \in \mathbb{Q}_{2}^{*}$ is a 2-adic unit $[7,1.7]$. Since -1 doesn't have this form, the 1-dimensional $G$-representation $H_{\mathbb{Q}_{p}}^{4}(B X)=Q H_{\mathbb{Q}_{p}}^{4}(B X)$ is the trivial one. Generators for the ring of invariant polynomials of the unique nonclassical 2-adic reflection group are [1, p. 101]

$$
\begin{aligned}
y_{8} & =x_{1} x_{2}^{3}+x_{2} x_{3}^{3}+x_{3} x_{1}^{3}, \\
y_{12} & =\operatorname{det}\left(\frac{\partial^{2} y_{8}}{\partial x_{i} \partial x_{j}}\right), \\
y_{28} & =\operatorname{det}\left(\begin{array}{cc}
\frac{\partial^{2} y_{8}}{\partial x_{i} \partial x_{j}} & \frac{\partial y_{12}}{\partial x_{i}} \\
\frac{\partial y_{12}}{\partial x_{j}} & 0
\end{array}\right),
\end{aligned}
$$

where the subscript on the variable $y$ denotes the dimension of the corresponding indecomposable cohomology class. Note that if an element of $N(W)$ takes $y_{8}$ to its opposite, then also $y_{12}$ is taken to its opposite but $y_{28}$ remains fixed. Thus any element of 2-power order in $N(W) / W$ must fix either $y_{8}$ or $y_{28}$ (considered as elements of $\left.H_{\mathbb{Q}_{p}}^{*}(B X)\right)$.

This proves the lemma for all almost simple $p$-compact groups.

Next suppose that $X$ is simply connected and nontrivial. Then there exist, by the splitting theorem [5], almost simple $p$-compact groups $X_{1}, \ldots, X_{n}$ with dual weight lattices $L_{1}, \ldots, L_{n}$ and Weyl groups $W_{1}, \ldots, W_{n}$ such that $X \cong X_{1} \times \cdots \times X_{n}$ and $L \cong L_{1} \times \cdots \times L_{n}$ as $W \cong W_{1} \times \cdots \times W_{n}$-modules. The efffect of $B g$ on $H_{\mathbb{Q}_{p}}^{*}(B X)=\bigotimes H_{\mathbb{Q}_{p}}^{*}\left(B X_{i}\right)$ has, cf. [8, 3.5], the form

$$
H_{\mathbb{Q}_{p}}^{*}(B g)=\left(A_{1} \otimes \cdots \otimes A_{n}\right) \circ \sigma
$$

where $A_{i}$ is an automorphism of $H_{\mathbb{Q}_{p}}^{*}\left(B X_{i}\right), 1 \leq i \leq n$, and $\sigma$ is a permutation within the isomorphism classes of these algebras. Hence

$$
Q H_{\mathbb{Q}_{p}}^{*}(B g)=\left(Q A_{1} \oplus \cdots \oplus Q A_{n}\right) \circ \sigma
$$

on $Q H_{\mathbb{Q}_{p}}^{*}(B X)=\bigoplus Q H_{\mathbb{Q}_{p}}^{*}\left(B X_{i}\right)$. There are now essentially two distinct cases to consider. Namely, the case where $\sigma$ is trivial and the case where $\sigma$ is a cyclic permutation of $p$-power order $>1$. The first case was treated above and in the second case, $Q H_{\mathbb{Q}_{p}}^{*}(B g)$ fixes the diagonal. Hence the fixed point vector space $Q H_{\mathbb{Q}_{p}}^{*}(B X)^{G}$ is nontrivial for any nontrivial simply connected $p$-compact group $X$.

Finally, up to isogeny any connected $p$-compact group has the form $X \times S[11$, 5.4 ] where $X$ is simply connected and $S$ is a $p$-compact torus and any automorphism 
is a product of an automorphism of $X$ with an automorphism of $S$ [10,4.3]. Hence

$$
Q H_{\mathbb{Q}_{p}}^{*}(B X \times B S)^{G} \cong Q H_{\mathbb{Q}_{p}}^{*}(B X)^{G} \oplus Q H_{\mathbb{Q}_{p}}^{*}(B S)^{G}
$$

is nontrivial if $X$ is nontrivial.

We conclude this note with the easy proof of Corollary 1.4.

Proof of Corollary 1.4. Let $\pi$ be the component group and $X$ the identity component of $Y$. The $p$-compact group extension

$$
X^{h G} \rightarrow C_{Y}(G) \rightarrow C_{\pi}(G)
$$

shows that $X^{h G}$ and $C_{Y}(G)$ have isomorphic identity components.

\section{REFERENCES}

1. D.J. Benson, Polynomial invariants of finite groups, London Mathematical Society Lecture Note Series, vol. 190, Cambridge University Press, Cambridge, 1994. MR 94j:13003

2. N. Bourbaki, Groupes et algèbres de Lie, Chp. 9, Masson, Paris, 1982. MR 84j:22001

3. W.G. Dwyer and C.W. Wilkerson, A new finite loop space at the prime 2, J. Amer. Math. Soc. 6 (1993), 37-64. MR 93d:55011

4. __ Homotopy fixed point methods for Lie groups and finite loop spaces, Ann. of Math. (2) 139 (1994), 395-442. MR 95e:55019

5. _ Product splittings for p-compact groups, Fund. Math. 147 (1995), 279-300. MR 96h:55005

6. R. Kane, The homology of Hopf spaces, North-Holland Mathematical Library, vol. 40, Elsevier Science Publishers B.V., Amsterdam-New York-Oxford-Tokyo, 1988. MR 90f:55018

7. J.M. Møller, The normalizer of the Weyl group, Math. Ann. 294 (1992), 59-80. MR 94b:55010

8. __ Completely reducible p-compact groups, The Čech Centennial. A conference on homotopy theory. Contemporary Mathematics, vol. 181 (Providence, Rhode Island) (M. Cenkl and H. Miller, eds.), American Mathematical Society, 1995, pp. 369-383. MR 97b:55020

9. __ Normalizers of maximal tori, Preprint, March 1995.

10. _ Rational isomorphisms of p-compact groups, Topology 35 (1996), 201-225. MR 97b:55019

11. J.M. Møller and D. Notbohm, Centers and finite coverings of finite loop spaces, J. Reine Angew. Math. 456 (1994), 99-133. MR 95j:55029

Department of Mathematics, University of Notre Dame, Notre Dame, Indiana 46556

E-mail address: William.G.Dwyer.1@nd.edu

Matematisk Institut, Universitetsparken 5, DK-2100 København Ø, Denmark

E-mail address: moller@math.ku.dk 